\title{
Alter
}

Revue de phénoménologie

\section{Chan-fai Cheung, Earthscape}

Zeta Books, 2013, 137 pages

Jean-Claude Gens

\section{OpenEdition}

Journals

Édition électronique

URL : http://journals.openedition.org/alter/331

DOI : 10.4000/alter.331

ISSN : 2558-7927

Éditeur :

Association ALTER, Archives Husserl (CNRS-UMR 8547)

\section{Édition imprimée}

Date de publication : 15 novembre 2014

Pagination : 313-318

ISBN : 978-2-9550449-0-2

ISSN : 1249-8947

Référence électronique

Jean-Claude Gens, "Chan-fai Cheung, Earthscape », Alter [En ligne], 22 | 2014, mis en ligne le 01 décembre 2017, consulté le 25 septembre 2020. URL : http://journals.openedition.org/alter/331 ; DOI : https://doi.org/10.4000/alter.331

Ce document a été généré automatiquement le 25 septembre 2020.

Revue Alter 


\title{
Chan-fai Cheung, Earthscape
}

\author{
Zeta Books, 2013, 137 pages \\ Jean-Claude Gens
}

\section{RÉFÉRENCE}

Chan-fai Cheung, Earthscape, précédé d'une introduction par Elmar Holenstein, suivie par deux essais de Jeff Malpas et de Kwok-yin Lau, Edwin Cheng Foundation Asian Centre for Phenomenology, The Chinese University of Hong Kong, et Bucarest, Zeta Books, 2013, 137 pages.

1 Essentiellement composé de photographies aériennes, Earthscape est néanmoins un ouvrage de phénoménologie. Après avoir commencé des études d'architecture, puis soutenu une thèse de doctorat en philosophie à Freiburg, son auteur, Chan-fai Cheung, qui a dirigé le département de philosophie de la Chinese University of Hong Kong, met en effet, en tant que photographe, en suspens nos appréhensions habituelles et, pour ainsi dire, naturelles de la terre; comme l'écrit Bernhard Waldenfels à propos du livre antérieur de photographies de Cheung intitulé Kaïros: Phenomenology and Photography ${ }^{1}$ : "Das alles ist ein "Doing phenomenology" ", pour reprendre le titre d'un livre de Herbert Spiegelberg. Si les photos de ce premier livre interrogent d'abord l'être des portes et des fenêtres, puis celui des sculptures funéraires, c'est dans la continuité de sa troisième et dernière partie intitulée « 30000 feet from above » que s'inscrit Earthscape.

2 Si pour un certain nombre de nos contemporains au moins, prendre l'avion est devenu quelque chose de suffisamment habituel pour ne plus susciter d'émotion particulière l'avion n'étant plus qu'un moyen de «déplacement" parmi d'autres dans lequel, comme dans un métro ou un train, on peut regarder un film, rédiger une conférence ou s'assoupir... -, pour Chan-fai Cheung en revanche, les voyages en avion n'ont rien perdu de leur magie lorsqu'ils nous donnent l'opportunité de percevoir notre terre sous un nouveau jour, et il a souvent exposé ses photos en particulier à Hong Kong (2008) et à Hawaï (2011). Bien que ce soit à travers une bonne centaine d'images qu'il cherche à partager l'étonnement éveillé en lui par un certain type de vues, Cheung ne renonce 
pas pour autant à l'explicitation conceptuelle du sens de sa pratique photographique : il s'en explique dans un bref essai introductif - comme il le faisait déjà dans Kaïros: Phenomenology and Photography -, et s'il ne recourt donc au discours langagier que de manière très réservée, il confie encore à trois de ses amis le soin d'interroger la signification de ces photographies aériennes.

On pourrait assurément interroger et discuter les thèses défendues par Cheung dès le premier moment de son essai introductif d'Earthscape, lorsque, invoquant Roland Barthes, il avance que le donné absolu est le fondement ontologique de la photographie, au sens où, à la différence de la peinture, « je ne peux jamais nier que la chose a eu lieu» (p. 10), Cheung ajoutant: "La photographie est plus proche de la phénoménologie que les autres arts. L'une et l'autre commencent avec la "chose même", le donné expérientiel absolu » (p. 10). Le second moment de l'essai de Cheung s'arrête à la spécificité de la photographie proprement aérienne en tant qu'art, et le troisième à l'idée même d'earthscape. Dans ce dernier moment, Chan-fai Cheung invite à concevoir la photographie aérienne non pas, comme on le fait généralement, comme un genre particulier de la photographie de paysage en général, mais à partir de la distinction, empruntée à Edward Casey, entre landscape et earthscape. Casey définit un scape comme

une vue limitée d'une scène particulière. C'est un lieu ou une région vue de quelque part par un corps qui regarde, quelqu'un qui est mû par un intérêt théorique, une curiosité, un souhait de mieux connaître le monde ambiant ${ }^{2}$.

Le landscape est ce qui se découvre toujours à un esprit dont le corps se tient sur la terre, ce qui se donne ainsi à percevoir ne se limitant pas à du visible puisqu'il peut être senti ou touché ; en revanche, "lorsque je suis assis derrière le hublot d'un avion, avance Cheung, la relation entre l'espace intérieur à l'avion et l'espace qui est extérieur à ce dernier est d'un genre spécifique ", puisque l'expérience de la terre au-dessus de laquelle je flotte dans un espace confiné se limite au visible et que la terre ne peut être touchée ; une rivière se limite ainsi à une ligne, un champ à une tache colorée..., ce qui se donne à voir ne se dessinant sur aucun horizon.

Dans sa postface d'Earthscape, Cheung rappelle une autre dimension de l'image photographique propre à l'earthscape : son caractère contingent ou aléatoire - un aléa que l'on retrouve par exemple aussi dans la photographie animalière ou le reportage de guerre - puisqu'elle implique que soient réunies toute une série de conditions comme le fait de disposer d'un siège devant un hublot qui ne donne pas sur l'aile de l'avion, une luminosité minimale qui fait défaut lors des vols de nuit, des conditions météorologiques comme l'absence de couverture nuageuse trop dense, et une attention constante, un caractère kaïrique déjà pointé par le titre du premier ouvrage de photographies de Cheung Kä̈ros: Phenomenology and Phenomenology.

Que nous donnent plus précisément à voir les photographies d'Earthscape? Notre chez nous, la terre dont nous sommes les habitants, nous les «terriens", mais sous un jour jusqu'alors inconnu. Cette terre n'est pas celle, primordiale, à partir de laquelle la perception est possible, cette arche originaire immobile, comme dit Husserl, qui constitue le sol, le foyer de tout percevoir, ou alors c'est, comme on le verra, cette terre dans ce qu'elle a néanmoins de vivant et d'inhumain ; ce n'est pas non plus la terre que nous habitons, celle qui, dans le monde de la vie, nous nourrit et nous enchante, celle qui - bien qu'elle nous soit familière - constitue malgré tout un autre dans la mesure où nous pouvons nous en éloigner ou en être éloigné de telle sorte que nous en avons alors 
la nostalgie. En d'autres termes, ces photos ne nous font pas voir ce que nous aurions jusqu'alors négligé de voir, comme, peut-être, les pommes peintes par Cézanne ou les visages peints par Marie Laurencin, mais des visages de la terre que nous n'avions jamais pu voir, qu'aucun oiseau n'a pu, et ne pourra, voir, et que seul l'éloignement d'un avion de ligne volant à trente mille pieds permet de voir. Que voit-on donc à cette distance?

7 Parfois - lorsque l'avion a décollé depuis peu ou est sur le point d'atterrir - des patchworks de champs traversés par les lignes de routes ou de fleuves et bordés par des monts inhabités plus sauvages (p.98), ou la géométrie d'espaces urbains; plus rarement, lorsque le ciel s'avère aussi important que la terre, ce sont autant des earthscapes que des skyscapes comme le suggère Malpas (p.3). Mais ces espaces sont généralement photographiés de si loin qu'ils perdent ce qui, de plus près, nous les fait apparaître comme familiers - espaces de mondes humains qui, dans cette distance, semblent appartenir à un monde étranger, voire chtonien ou pré-humain et potentiellement inquiétant. Autrement dit, la distance à partir de laquelle la terre est vue est telle qu'elle nous rend cette terre étrangère.

D'autres fois, ce sont des massifs montagneux ou des chaines montagneuses qui s'étirent les unes après les autres pour former comme des vagues successives dans une mer de montagnes prises dans des brumes de sorte que ces vues s'avèrent avoir un air de parenté non tant avec des peintures comme le Voyageur contemplant une mer de nuages de Caspar David Friedrich où c'est encore une figure humaine qui domine cette mer, que, comme on le verra, avec des peintures chinoises ; à l'encontre de l'idée que nous nous faisons le plus souvent de la terre, celle qui nous fut inculquée et selon laquelle elle est un corps minéral et inerte revêtu seulement ici ou là d'une fine pellicule de vivants, même lorsqu'on «reconnaît» leurs formes, ces massifs apparaissent sous un jour radicalement neuf, par exemple lorsque leurs formes ressemblent à des peaux craquelées (p. 39, 122), et plus encore lorsque leurs sinuosités leur donnent des allures animales (p. 79, 86) analogues à celle, par exemple, d'une anguille. Une telle hauteur permet à Cheung de mettre en évidence les «structures de la terre » dit Waldenfels ; « nervures » serait peut-être plus adéquat au regard de la vie, quasi organique, qui semble habiter ces formes - des formations fluides qui pourraient être celles de réseaux de neurones (p. 17) ou des formations de laves liquides, alors qu'il ne s'agit pas de mers ni de volcans. Mais le plus souvent on ne reconnaît rien : de pures et improbables formes colorées, des surfaces sans profondeur, peu différentes de celles auxquelles nous a habitué l'art dit « abstrait » ou de pures lumières.

9 La singularité des images d'Earthscape ressort d'autant mieux qu'on les compare à celles, célèbres, de La terre vue du ciel de Yann Arthus-Bertrand. Bien qu'on puisse trouver dans La terre vue du ciel quelques images comparables à celles d'Earthscape ${ }^{3}$, ces images restent très rares dans la mesure où, comme le remarque Cheung, ArthusBertrand vise explicitement à susciter un nouvel intérêt, un nouveau respect pour la fragilité de la planète en tant qu'elle constitue notre environnement (p. 11), à suggérer qu'il nous faudrait transformer la manière dont nous exploitons et consommons ses ressources. Arthus-Bertrand photographie donc la terre, certes vue du ciel, mais à une altitude qui permet encore de reconnaître des formes, de reconnaître la terre en tant qu'elle constitue notre chez nous (l'altitude à laquelle volent une montgolfière ou un deltaplane, et, le plus souvent ici, un hélicoptère qui, comme le dit Arthus-Bertrand, est susceptible d'être commandé "comme une grue », ce qui réduit d'autant le caractère 
kaïrique de ces photos). Autrement dit, cette altitude, c'est celle qui permet d'accomplir l'intention indiquée par le sous-titre de La terre vue du ciel : réaliser un "portrait » de notre terre natale, et l'on ne peut faire de portrait que d'une forme précise et non d'une entité informelle et mouvante. On comprend que les images de ce livre aient chacune, en tant que portrait, un titre indiquant un lieu dont les coordonnées géographiques précisent encore plus l'identité de la chose photographiée. Cheung préserve au contraire l'anonymat des étranges espaces qu'il photographie en se gardant bien de leur donner des titres; ne sont en effet mentionnées en fin de volume que la nature et l'année des vols qui, entre 2004 et 2011, l'ont conduit aux États-Unis, à Dubaï, en Europe ou en Chine continentale.

10 Mais c'est souvent moins le contraste avec d'autres genres de photographies aériennes que le rapprochement avec des œuvres picturales qui éclaire le propre des images, pourtant jamais vues, d'Earthscape. Je pense, par exemple, aux formes de certaines de ces images qui ressemblent à ces volutes que l'on trouve, figurant des nuages, sur les tankas tibétains, ou, comme le remarque aussi très justement Holenstein, à des peintures comme La naissance du printemps du peintre Song du XI siècle Guō Xī (郭熙) ; ce que ce dernier note dans son célèbre traité intitulé Monts et rivières à propos de ce que la peinture doit donner à percevoir vaudrait ainsi pour ce que cherchent à saisir les images photographiques de Cheung:

Les nuages et les vapeurs de paysages réels ne sont pas les mêmes pour les quatre saisons. Au printemps, ils sont clairs et diffus, en été riches et denses, en automne dispersés et fins, en hiver sombres et solitaires. Quand de tels effets peuvent être vus en peinture, les nuages et les vapeurs ont un air de vie. La brume autour des montagnes n'est pas la même selon les quatre saisons. La montagne au printemps est claire et séduisante comme si elle souriait. La montagne en été a une couleur bleu-vert qui semble diffusée dessus. Les montagnes en automne sont lumineuses et belles comme si elles étaient fraîchement peintes. Les montagnes en hiver sont tristes et tranquilles comme si elles dormaient

11 - sauf que la longue familiarité avec la chose même des vapeurs et des nuages dans les montagnes à laquelle doit parvenir le peintre n'est pas du même ordre que l'attention à laquelle le photographe doit s'exercer pour espérer saisir ce qui ne lui est donné que par le kaïros.

12 Au-delà donc des vues peu nombreuses qui nous reconduisent à notre terre natale dans ce qu'elle a de familier et de fragile, l'epokhè des perceptions habituelles que nous avons d'elle, l'estrangement induit par la vision à une altitude de 30000 pieds, laisse encore entrevoir une terre plus obscure, une terre pré-humaine dans ce qu'elle a d'immuable ou d'éternel - celle qui constitue le soubassement de l'historicité célébrée par la pensée moderne, celle à l'attention de laquelle nous appelle Karl Löwith -, mais dont la nature est difficile à saisir, car équivoque ou multiple; ce qu'elle a d'immuable, c'est son caractère changeant et mobile en tant qu'elle est parcourue par des lignes de forces, ou, pour le dire en des termes qui conviendraient aussi à La naissance du printemps, en tant qu'elle est habitée par le qi (气); autrement dit, et pour reprendre les termes de Cahill décrivant les paysages de Guō Xī, ce qui caractérise ces formes, c'est «la turbulence d'un monde en mouvement ", ce sont " les formes terrestres gonflées au-delà de toute vraisemblance par le flux qui les anime, [qui] se fondent et s'interpénètrent tels les organes d'un corps immense $»^{4}$. Mais, comme on l'a brièvement mentionné, ces formes qui semblent en perpétuelles transformations laissent parfois aussi sourdre la dimension chtonienne et inquiétante de la terre, une terre dont il faudrait donc au 
moins parler au pluriel - les terres - en préservant l'énigme de cela qui ne nous habite pas moins que nous ne l'habitons.

\section{NOTES}

1. Chan-fai Cheung, Kaïros: Phenomenology and Photography with Essays by Hans Rainer Sepp and Kwok-ying Lau, Bucarest, Zeta Books, 2010.

2. Edward Casey, "Mapping the Earth in Works of Art ", in Bruce W. Folts et Robert Frodeman, éd., Rethinking Nature: Essays in Environmental Philosophy, Bloomington, Indiana University Press, 2004, p. 264.

3. Voir par exemple Flancs enneigés du volcan Kronotskaya, Kamtchatka, ou Glaces sculptées par le vent au sommet du Mont Discovery, Antarctique, in Y. Arthus-Bertrand, La terre vue du ciel. Portrait aérien de la planète (1999), Paris, Éditions de La Martinière, 2005 (435 pages), respectivement pp. 414 sq. et $424 \mathrm{sq}$.

4. James Cahill, La peinture chinoise, trad. Y. Rivière, Genève, Skira, 1995, p. 35. Sur La naissance du printemps, voir aussi la présentation par Y. Escande de Guo Ruoxu, Notes sur ce que j'ai vu et entendu en peinture, Bruxelles, La lettre volée, 1994, pp. 35 sq. 